\title{
DEVELOPMENT OF A MECHANICAL TESTING AND LOADING SYSTEM FOR TRABECULAR BONE STUDIES FOR LONG TERM CULTURE
}

\author{
D.B. Jones ${ }^{1 *}$, E. Broeckmann ${ }^{1}$, T. Pohl ${ }^{1}$ and E.L. Smith ${ }^{2}$
}

\author{
${ }^{1}$ Department of Experimental Orthopaedics and Biomechanics, University of Marburg, Germany \\ ${ }^{2}$ Biogerontology Laboratory, Department of Population Health Sciences, University of Wisconsin-Medical School, \\ Madison, WI, USA
}

\begin{abstract}
A highly accurate $( \pm 3 \%)$ mechanical loading and measurement system combined with a trabecular bone diffusion culture-loading chamber has been developed, which provides the ability to study trabecular bone (and possibly) cartilage under controlled culture and loading conditions over long periods of time. The loading device has been designed to work in two main modes, either to apply a specific compressive strain to a trabecular bone cylinder or to apply a specific force and measure the resulting deformation. Presently, precisely machined bone cylinders can be loaded at frequencies between $0.1 \mathrm{~Hz}$ to $50 \mathrm{~Hz}$ and amplitudes over 7,000 $\mu$ e. The system allows accurate measurement of many mechanical properties of the tissue in real time, including visco-elastic properties. This paper describes the technical components, reproducibility, precision, and the calibration procedures of the loading system. Data on long term culture and mechanical responses to different loading patterns will be published separately.
\end{abstract}

Key Words: Trabecular bone diffusion, static and dynamic mechanical loading, biological stimulation, stiffness-measurements, Young's Modulus.

* Address for correspondence:

David B. Jones

Department of Experimental Orthopaedics and Biomechanics

University of Marburg

Baldinger Str.

D-35033 Marburg, Germany

Phone number: +49-6421-286-6484

FAX number: +49-6421-286-8981

E-mail: jones@med.uni-marburg.de

\section{Introduction}

Numerous in vivo and in vitro models have been developed to investigate the changes in bone metabolism and bone morphological changes, induced by mechanical loading. While significant progress has been made, the transducing mechanisms behind mechano-sensing with regard to the sensing system and which cell type or types are responding remain unknown. These models utilize cell culture (Yeh and Rodan, 1984; Jones et al., 1995, Zaman et al., 1997) organ culture (Rodan et al., 1975; Cheng et al., 1996) or the whole organism (Rubin and Lanyon, 1987; Chow et al., 1998; Ahkter et al., 2002; Rubin et al., 2002). Cells in bioreactors have also been investigated (for example: Botchway et al., 2001). None of these in vitro models reproduce the amplitudes, direction, mode or frequencies occurring in the loaded tissue in vivo due to the highly complex nature of physiological loading. Another method is to cut portions of bone, such as trabecular bone, to shape, which makes loading more quantitative (e.g. El Haj et al., 1990) but do not allow good tissue physiology.

Bone cells in vitro may appear to respond to physical stimuli by a number of mechanisms, but they are not grown under physiological conditions and the results may reflect a number of culture artefacts rather than a real physiological response. Organ cultures do not have a blood supply and quickly degenerate a few hundred micrometers from the surface. Application of mechanical loads is difficult to quantify in this model. Animal experiments are expensive and difficult to perform. This is perhaps why it has been noticed that bone cells and bone organ culture appear to respond differently to dynamic strain (Rawlinson et al., 1995) as well as the strain in the animal.

Current bone testing and loading systems that are used to evaluate and test living tissue use hydraulics or a variety of levers, cams, and screws do not have the capability to provide tightly controlled real-time measurements of force and deformation under dynamic loading conditions, especially at frequencies above $10 \mathrm{~Hz}$. The quantification of forces is also difficult, especially in organ culture models. Other loading systems, using segments of bone or whole bones, estimate or calculate strain distributions from a limited number of measurements on specimens using strain gauges.

A precise and quantifiable loading system is necessary in order to apply well-defined mechanical perturbations to specimens that can vary widely in their material and structural properties. We are aware that although the compressions and forces may be applied with high precision, the deformations within the tissue that 


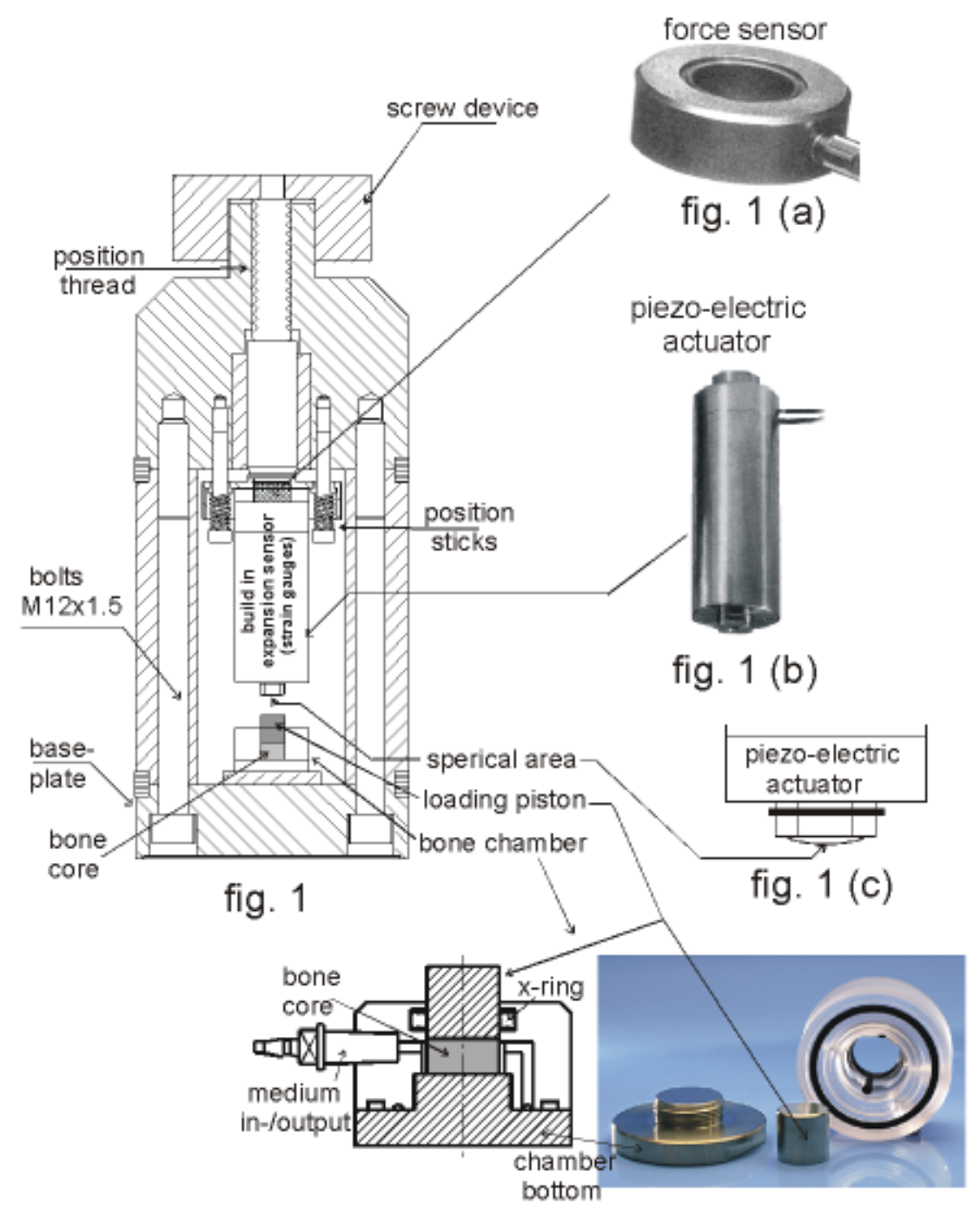

fig. 1 (d)

Figure 1: The housing of the loading device is made of three cylindrical sections, 15 centimetres in diameter, bolted together with six hardened steel bolts. The front of the housing has an opening for placing the culture bone chamber in position for loading. The chamber is centrally positioned by mechanical stops in the rear of the opening assuring that the chamber is directly centred under the piezo-electric actuator. At the top of the housing is a screw mechanism to raise and lower the actuator onto the bone chamber. It is also used to induce a mechanical preload on the bone cores by hand. Sensors and actuators are shown in the figures on the right: (a): force sensor, (b): piezo-electric actuator with a build-in strain gauge sensor to measure the actuator's expansion, (d): bone chamber. All these components are placed on-axis in the middle of the loading device.

result are highly complex.

The purpose of this paper is to describe the technical components, reproducibility, precision, and the calibration procedures of the loading system (Figure 1) the culture loading chamber and the associated technology will be presented in detail elsewhere. In preliminary studies trabecular bone cores have been maintained viable during non-loading (Smith et al., submitted for publication) and loading conditions in the culture loading chambers for 28 day (bovine) (Smith. et al., 2000) and 49 days (human femur). (Smith et al., 2001). Our bone samples were obtained from bovine and human trabecular bone tissue and prepared under sterile environments in a laboratory of the clinic of the University of Marburg.

\section{The Loading System}

The loading system (LS) (Figure 1) consists of a resistive frame of steel bolts with stainless steel end caps and an aluminium housing. A screw mechanism raises and lowers the load sensor - piezo-electric actuator unit with the load sensor type $9011 \mathrm{~A}$, made by KISTLER ${ }^{\mathrm{TM}}$ (Winterthur, Switzerland) (Figure 1a) and with the piezoelectric actuator (PZA) type P-239K078 (including strain gauges) made by Physik Instrumente ${ }^{\mathrm{TM}}$,(PI) (Karlsruhe, Germany) (Figure 1b) within the aluminium housing. Using the screw mechanism a small static preload may be applied to a sample. Any further desired preloads are applied by the PZA. This brings the interfacing surfaces of the machine and the machine-bone interfaces into contact (see below). 


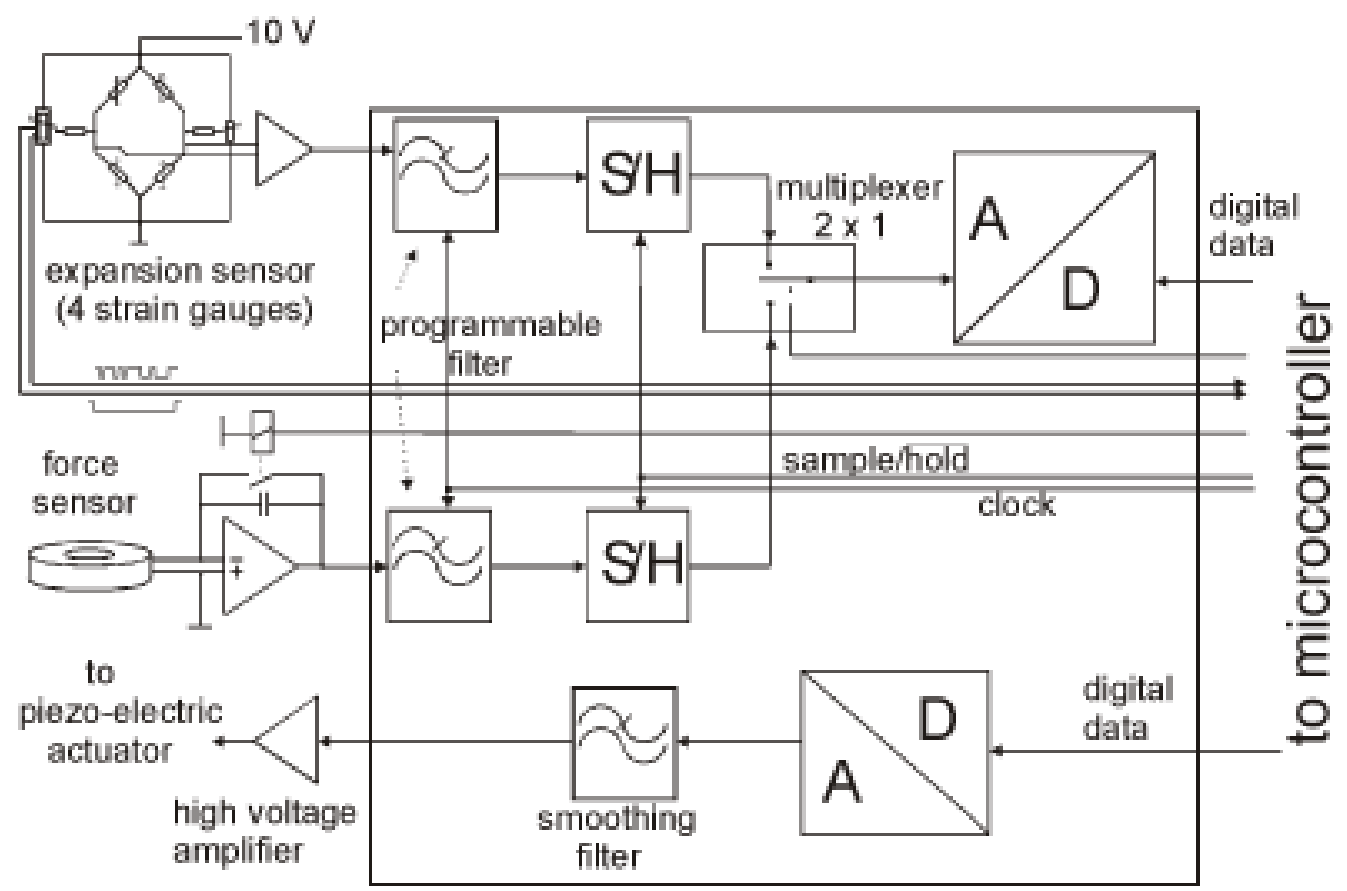

Figure 2: The Data Acquisition Module contains two identical programmable low pass filters for noise reduction. The two sample/hold-amplifiers $[\mathrm{S} / \mathrm{H}]$ have the control line in common to provide simultaneous sampling of force- and expansion signal. By use of a 2 to 1 multiplexer this board needs only one analogue-digitalconverter $[\mathrm{A} / \mathrm{D}]$. This figure also shows the principle circuit diagram of a charge amplifier (middle, left), which is used to amplify the force sensor's response. The amplifier works as a current integrator presenting an output voltage linear to input charge. The relay is used to set the output to zero thereby removing residual charge. The expansion sensor is shown in the top left corner. This is a four-element strain gauge sensor. The gauges are configured as a Wheatstone bridge. An instrumentation amplifier intensifies the cross voltage of the bridge by a factor of approximately 500 .

A high voltage amplifier (PI type E-420.00), whose input is connected to the analogue output of a microcontroller circuit (in house design), activates the PZA. Together with a WINDOWS based user interface program it is possible to apply a quantifiable force or a quantifiable deformation of the bone core in the culture-loading chamber. The microcontroller and associated electronic system and sensors work independently of the PC's operating system and record and store real-time measurements of both PZA's expansion (from strain gauges) and force (load cell). The LS is currently configured to load trabecular bone cores 5 or $10 \mathrm{~mm}$ in height and $10 \mathrm{~mm}$ or $12 \mathrm{~mm}$ in diameter, that are housed in a culture loading chamber (Figure 1d).

\section{Piezo-Electric Actuator/Expansion Sensor}

The stiffness of the PZA is $128 \mathrm{~N} / \mu \mathrm{m}$. It can apply forces of up to $4,500 \mathrm{~N}$. The maximum expansion depends on the load application. The nominal maximum expansion under no load conditions is typically $60 \mu \mathrm{m}$. These types of actuators show small hysteresis effects. Their expansion at constant drive voltage also depends slightly on previous expansions. In dynamic operation the amplitude of expansion depends on the operation frequency in combination to resonant frequency of the PZA in connection to the oscillating effective mass, visco-elasticity and stiffness of the bone or other material to be loaded. Furthermore the PZA's expansion does not follow the high voltage amplifier's input signal congruently at frequencies higher than 50 $\mathrm{Hz}$. Frequencies above $50 \mathrm{~Hz}$ cause an amplitude drop, a phase shift, and the actuator's dynamic response lessens resulting in a distortion of the actuator's displacement waveform. This is a result of the power range and the dynamic response of the commercial amplifier. Thus the expansion of the PZA cannot be derived from the piezo's drive voltage directly. The measurement of expansion requires a separate expansion sensor, which may be configured with strain gauge sensors or capacitive coupled sensors to determine the precise $( \pm 20 \mathrm{~nm}$ or \pm 1 nm respectively) actuator's expansion. We used the PZA equipped with strain gauges, configured as a Wheatstone bridge as an established standard solution in strain measurements.

The lower contact surface of the PZA is covered with a sapphire plano-convex lens (VICTOR KYBURZ AG, Safnern, $\mathrm{CH}$ ) with a radius of $50.8 \mathrm{~mm}$ ( 2 inch) (Figure 1c). The spherical nature of the contact surface allows for loading adaptation of the system to slightly non-parallel bone sample surfaces and prevents bending stress within the crystal stack and in case there is a wedgeshaped gap between the PZA and the loading piston. The design of the culture loading chamber (Figure 1d), in which the loading piston is sealed with an ' $X$ ' ring ('Quad ring' Busak \& Shamban S.A. Crissier, CH No. 


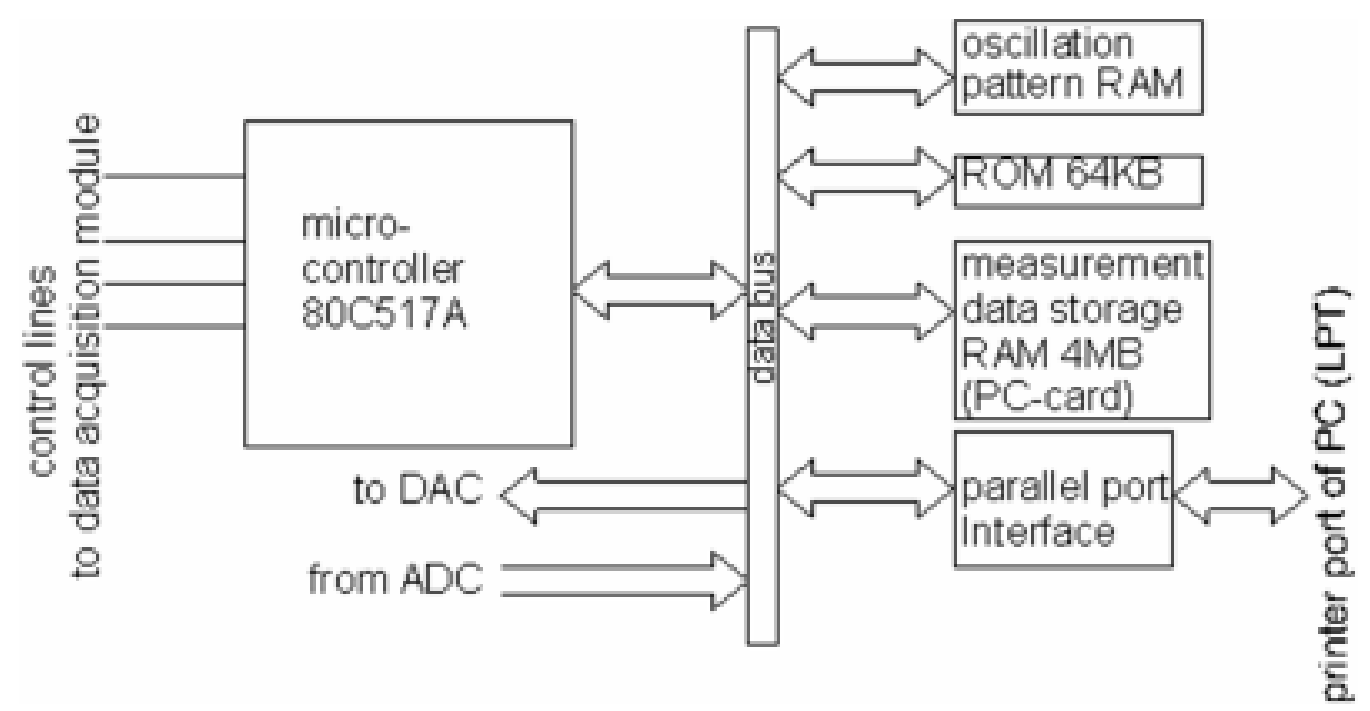

Figure 3: This figure shows the microcontroller and its 8-bit bus environment: memories (RAM: data, ROM: program), the parallel port interface for data communication with the host computer, bus interface to digitalanalogue-converter [DAC] and analogue-digital-converter [ADC].

QRAR4111A-E7509) also allows some accommodation of non-parallelity of up to $50 \mu \mathrm{m}$ (the theoretic limit due to the bone chamber's geometric tolerances). However the precision of the sample is kept within $\pm 2 \mu \mathrm{m}$ in roughness and parallelity by using precise histological diamond annular and band saws to minimize non-linearities in the measurement data.

\section{Electronic Modules}

The PZA is driven by a High Voltage Piezo-Actuator (HVPZT) Amplifier (Physik Instrumente ${ }^{\mathrm{TM}}$, (PI) type E420.00). The output voltage range is 0 to -1000 volts. The high voltage amplifier (bottom left in Figure 2) has its own power supply, and is switched on/off by the microcontroller of the electronic system (see below).

\section{Data Acquisition Module}

This module contains sensor amplifiers, a digital-analogue-converter (DAC), an analogue-digital-converter (ADC), filters and sample-hold circuits and was designed and built in-house. Figure 2 shows all parts of this module in a box together with sensors, sensor amplifiers and the HVPZT-amplifier. The outputs of the sensor amplifiers are passed to switched capacitor low pass filters for noise reduction. The common cut-off frequency of the filters is set by the microcontroller. Sample/hold amplifiers allow exact simultaneously sampling of the analogue signals, which represent values of force and PZA's expansion.

\section{Microcontroller Module}

To avoid complications with critical timing in multitasking operating systems on a host personal computer, a separate microcontroller circuit was designed within the ba- sic electronics of the loading system. This is designed for real-time data collection from force and expansion sensor even at high sampling frequencies up to several $\mathrm{kHz}$. The module includes a microcontroller board (type "BDEmodule 517-light", manufacturer: PHYTEC ${ }^{\text {TM }}$, Germany), which carries a PC-card memory and a microcontroller of type 80C517 (Siemens ${ }^{\mathrm{TM}}$ ), comprehending the established Intel 8051 CPU-core.

The memory organization of the microcontroller module is shown in Figure 3. Data is collected with 12 bits of resolution. The oscillation-pattern RAM is provided to store downloaded data, for instance a dynamic stimulation pattern, whereas the PC-card memory is used to store values of force and expansion (sensor data) of a measurement. The microcontroller is connected with the printer port of a PC or notebook for bi-directional data communication. This line is faster than a serial line such as RS232C. Measurement procedures are fully controlled by the microcontroller whereas the PC's control program may handle other tasks.

\section{Force Sensor/Amplifier}

A piezo force sensor, made by KISTLER ${ }^{\mathrm{TM}}$ (Figure 1a), with a stiffness of $1.8 \mathrm{kN} / \mu \mathrm{m}$ was used to measure force application to the bone cores. The response to force of the sensor is an electric charge linear to the magnitude of the force applied to the sensor. Simple circuits with the sensor and charge amplifier (Figure 2, middle left) do not always measure absolute values. The general nature of a piezo-electric sensor is that some residual charge will be maintained even without load resulting in an offset error at the amplifier's output. Thus the system's control program has the feature, which sets the force amplifier into a reset mode (all residual charge dropped) and an operate mode (amplifier ready to work), which is turned on just before starting a measurement. 


\section{Expansion Amplifier}

The expansion sensor's cross voltage is passed to an instrumentation amplifier, shown in Figure 2 (top, left). The gain of this amplifier is about 500. There are two potentiometers for rough and fine zero-adjustment: the first potentiometer (Figure 2, to the right of the bridge) is adjusted during final manufactory control, the second (to the left of the bridge) is a digitally driven potentiometer controlled by the microcontroller.

\section{Quasi-Static and Dynamic Loading}

To measure the stiffness of bone or reference bodies (see below), we used quasi-static loading where the PZA's drive voltage is gradually increased by small incremental steps over approximately 30 seconds with the force and displacement measured simultaneously and data collected and stored. A low-pass filter smoothes the PZA drive voltage signal to avoid dynamic stimulation. Furthermore the PZA is released slowly (10 seconds) and smoothly applying a falling edge in form of half a cosine-period (Figure 4).

The apparent stiffness $(\mathrm{k})$ is calculated by taking the reciprocal slope $(\mathrm{s})$ of a linear regression line $\left(\mathrm{k}=\mathrm{s}^{-1}\right)$ over a certain selectable force range $\left[\mathrm{f}_{\mathrm{a}}, \mathrm{f}_{\mathrm{b}}\right]$ with the profit of noise reduction by a factor of $n^{1 / 2}$, where $n$ is the number of samples taken for linear regression. In the case of nonlinear materials the user has to select an interval on the force axis, where the curve looks linear (Figure 5). Our program has the feature to store the limits $\mathrm{f}_{\mathrm{a}}, \mathrm{f}_{\mathrm{b}}$ of the interval associated with the label of a given bone core and automatically restores the same force interval $\left[f_{a} f_{b}\right]$ whenever the sample is re-measured.

The program presents the stiffness in terms of a Young's modulus (E, in MPa or GPa units) using cylindrical specimen dimensions, the radius (r) and height (h), for normalization to extract a value, which does not de-

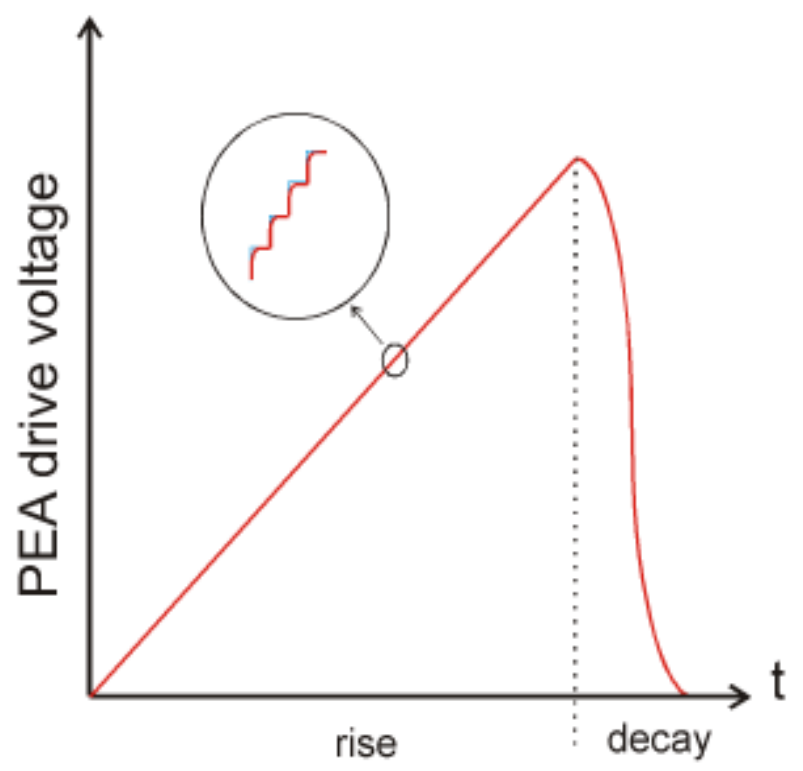

Figure 4: In quasi-static load the drive voltage to the piezo-electric actuator is increased by small smoothed incremental steps. The falling edge is realized in form of a cosine-edge to avoid dynamic stimulation of bone.

pend on the geometric dimensions of the body:

$$
\mathrm{E}=\mathrm{k} \cdot \frac{\mathrm{h}}{\pi \mathrm{r}^{2}}
$$

In this way we obtain a value, which can be practically associated with mechanical properties of other material.

The purpose of this measurement however is not to derive 'real' measurements of the bone stiffness, since cutting the cylinders of trabecular bone out of their context

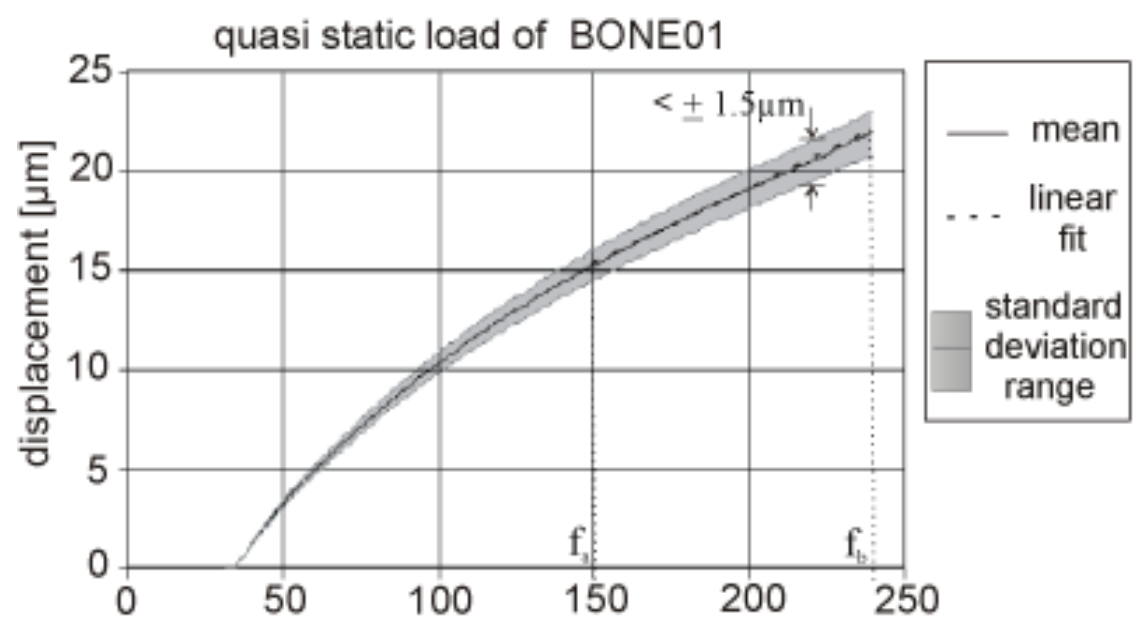

Figure 5: This figure presents an example of data of a quasi-static load on bone. While the piezo-electric actuator's expansion is gradually increased, force and displacement are measured simultaneously. This figure also shows the standard deviation borders of a number of repetitions. The thick line on the right half of the figure is a linear-fit line in the range of $f_{a}, f_{b}$. The reciprocal value of the slope is taken to obtain the stiffness of the bone sample. 


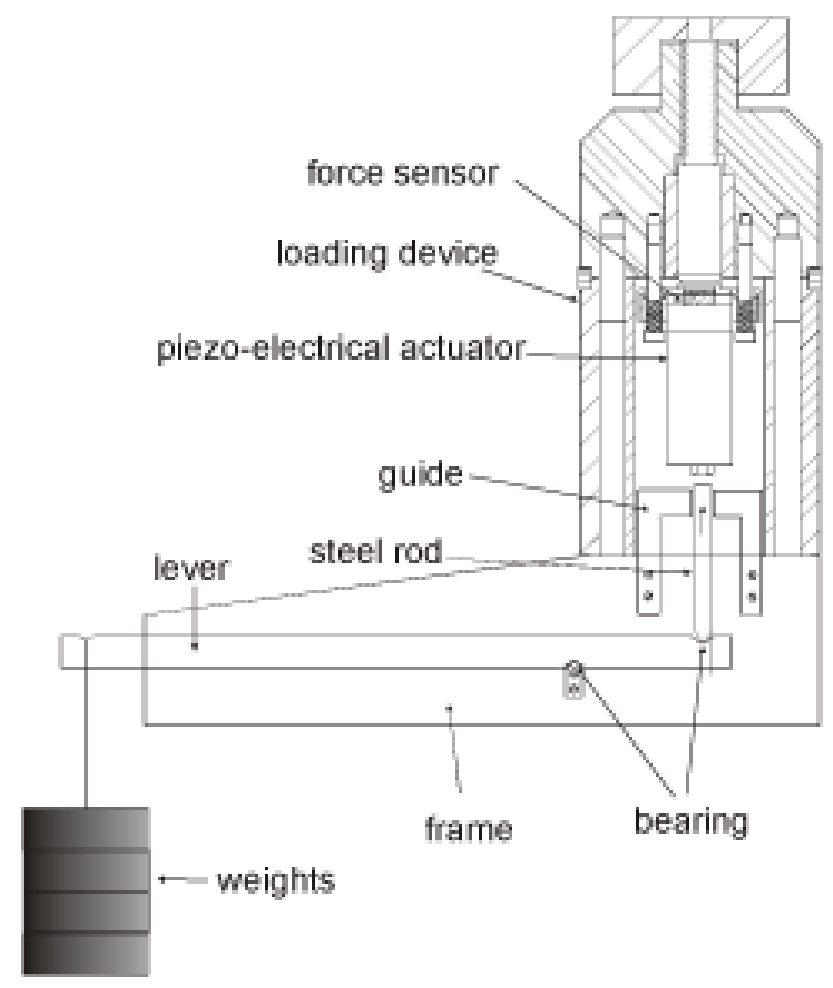

Figure 6: The force calibration device is mounted at the bottom of the loading device after having removed the base plate. The specially machined rod of stainless steel shown here applies force to the bottom of the piezoelectrical actuator. This amount of force also acts on the force sensor because all parts are placed on the common vertical centre axis and there is no force-bypass. The lever is used to amplify the force generated by the number of weights applied at the left.

removes many struts and supports. The mechanical stiffness measurements thus gained may not be extrapolated to the whole bone. The purpose is to obtain a precise value, which is used for comparison for subsequent treatments i.e. has the sample increased or lost stiffness and how this is related to biochemical changes or histological or structural changes revealed by $\mu \mathrm{CT}$ imaging. Analysis of the structure revealed by $\mu \mathrm{CT}$ may be used in future to use in finite element modeling. (For $\mu \mathrm{CT}$ analysis we use a modified bone chamber, where the metallic loading piston and the chamber bottom are replaced by non-metallic sapphire cylinders.)

In dynamic loading various oscillation pattern such as standard sinusoidal-, triangular-, square-signals and complex waveforms can be applied on a bone sample. Data of force and PZA's expansion can be measured simultaneously during loading. The build-in function generator also accepts input of a series of numbers to generate specific designed waveforms. With this feature we have imported waveforms of normal walking and jumping from a force platform and apply these physiological waveforms in our current bone loading program.

\section{Mechanical Calibration}

\section{Calibration of Force}

The force sensor - located at the top of the PZA (see Figure 1a) - was calibrated at the manufacturer, however the assembly screw, which applies a significant pre-force to compress the surfaces together, always shunts part of the force and therefore the sensor must be calibrated again after installation to determine the final sensitivity of the completed measuring setup. To set the charge amplifier to $1,500 \mathrm{~N}$ full scale, we switched the pre-scaler to a suitable sub-range and adjusted the gain-potentiometer of the amplifier to 10 volts (maximum) at 1,500 Newton.

We determined a scale factor, which includes the sensor's sensitivity and the gain-factors of all other electronic parts. A simple force calibration device was designed, as shown in Figure 6. Calibrated weights were placed on the left end of a horizontal lever. The lever is a 'wedge on mortise' to ensure a fixed and perfectly determined rotation axis and has a geometric ratio of 12:1. With seven different weights and linear regression of digital numbers (analogue-digital converter output, Figure 2) produced a correlation coefficient of 0.999942 . The standard deviation of force variations from linear fit leads to a relative full-scale error of $0.22 \%$.

\section{Calibration of Expansion - Displacement}

The following methods were developed to determine the displacement in the bone or of any other material under the loading piston (see Figure 1d).

The culture loading chambers were designed to accept a trabecular bone cores that are 5 or $10 \mathrm{~mm}$ in height and $10-12 \mathrm{~mm}$ in diameter. In preliminary measurements 

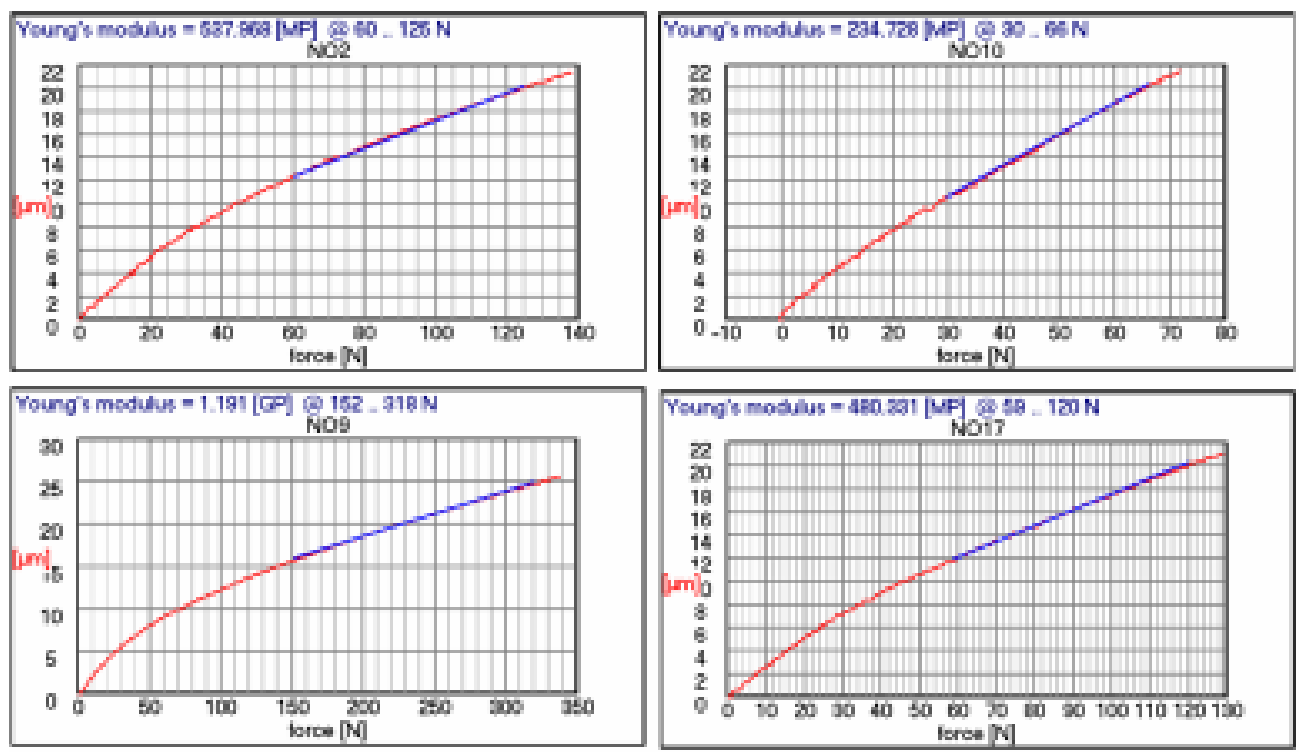

Figure 7: Examples of quasi-static load measurements: Stiffness measurement of different bone-cores

Figures $9 \mathrm{a} / 9 \mathrm{~b}$ show expansion versus force and displacement respectively. All curves show mean values over 8 repetitions. When the expansion of the piezo-electric actuator is measured without any load (interferometer touches the PZA-bottom directly) then we obtain a vertical straight line directly covering the y-axis (not shown on the plot), because there is no force.

We transferred the fundamental triplet data on these reference bodies into a look-up table. It is a two-dimensional table, which is addressed by the digital values of force and PZA's expansion in the system's control program. The expansion data are not of final interest; they are only intermediate values that are used to address the look-up table to determine the sample's displacement. Figure 10 shows the same curves as shown in Figure 9a/ $9 \mathrm{~b}$ but in a three-dimensional plot based on the forceexpansion-plane. All cells of the complete (f,x)-plane are filled by linear interpolation between the measured curves. To estimate the total error in displacement we analyzed the row data of our calibration measurements and found that standard deviations (not shown in Figure 9a/9b) increased with increasing in force and stiffness. The standard deviations are greater than the resolution units of the sensors after conversion to digital numbers: expansion sensor: » $\pm 0.015 \mu \mathrm{m}$, interferometer: $\pm 0.01 \mu \mathrm{m}$. We assume that the precision/roughness of the reference bodies' surfaces is responsible for this. Table 2 shows typical values below and above $400 \mathrm{~N}$ :

A Young's modulus greater than $1000 \mathrm{MPa}$ can cause errors up to $2 \mu \mathrm{m}$, but this is above the design limit of our system. Smallest variations occur in the range $100-$ $1000 \mathrm{MPa}$ and $<400 \mathrm{~N}$, whereas below $100 \mathrm{MPa}$ errors are increasing again. Our specific application involves low values of force and an analysis of our raw data shows that the noise in force measurement (resolution unit is » \pm 0.4 $\mathrm{N})$ is the dominant part.

Our assumption is that the standard deviation of our measurements data stands for total displacement-errors, which are reduced to some typical values shown in table 2. These errors are lower than the mechanical precision of the surfaces of the reference bodies.

\section{Control of Quality and Stability}

The loading system has been specifically configured to induce quantifiable loads and deformation to trabecular bone tissue and measure the physical characteristics of the bone tissue to a variety of perturbations of force, deformation, waveforms, frequencies and duration. However, the loading system must be coupled with the specially designed culture-loading chamber for the maintenance of long-term bone viability and the ability to evaluate the induced perturbations at a single time point or to monitor bone for 30 to 47 days. In order to have a useful tool for the study of bone both precision and reproducibility must be quantified and demonstrated in both reference bodies and bone tissue. The very nature of bone heterogeneity results in greater variation in measurement due just to the physical properties of bone compared to the homogeneity of the metallic reference bodies.

Three areas were investigated relative to long-term reproducibility of the reference bodies and apparent bone stiffness over 20 to 28 days. The physical characteristics of both the bone and chamber surfaces and the placement of the reference were evaluated after the calibration of the loading system. To study the long-term responsibility and precision of the loading system a quasi-static loading program was used for both bone cores and reference bodies. For controls, none dynamically stimulated bone cores were measured every 4 days applying a constant force in quasistatic loading, using $25 \mu \mathrm{m}$ of compression in order to determine their apparent stiffness and the reproducibility of deformation over a 20-day period. The standard deviations of deformation are in the range of $0.6-1.3$ micrometer (Figure 11) but we also see a tendency of a small drop in stiffness about $1.5 \%$. This small drop is consistent with 


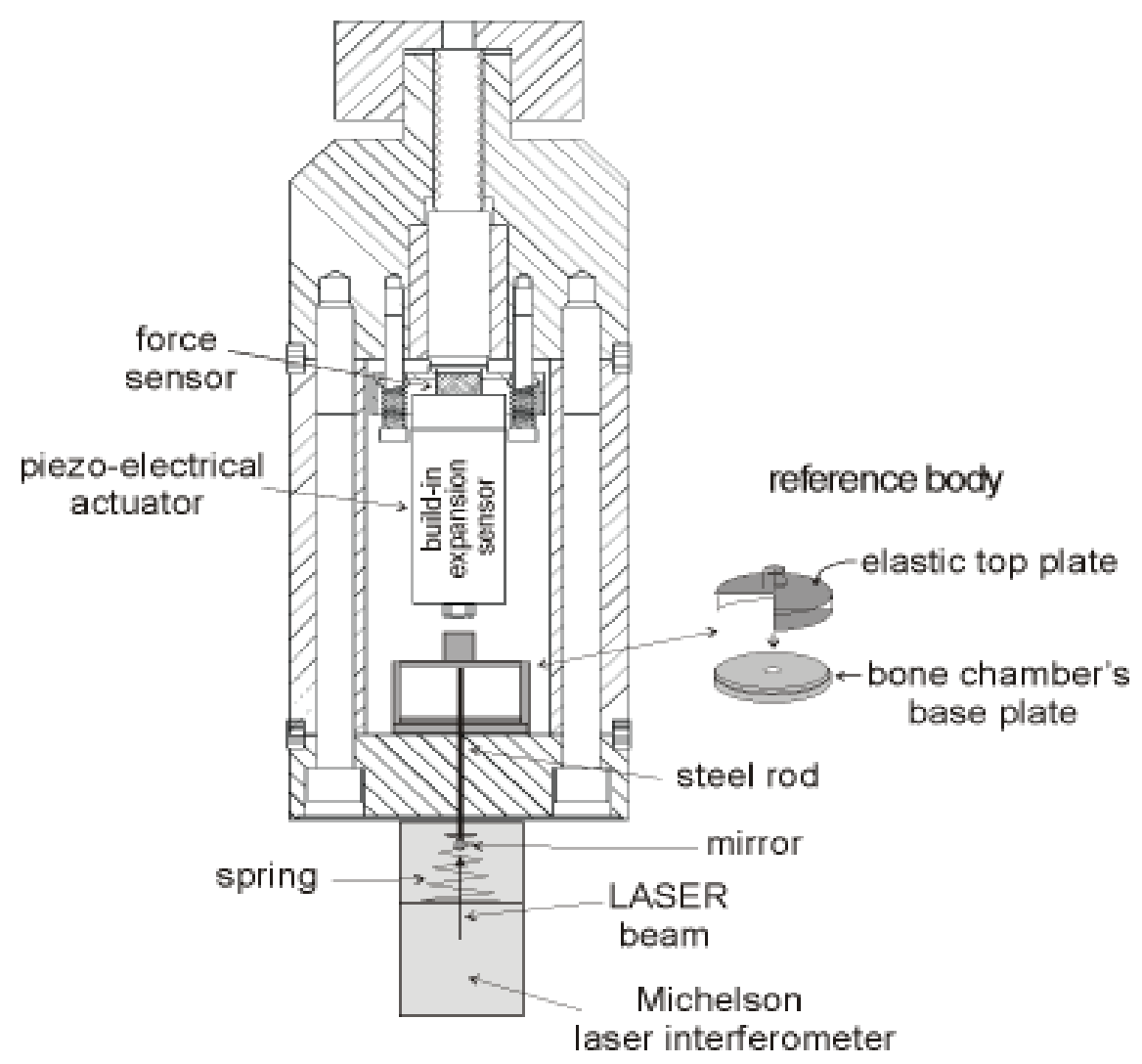

Figure 8: This figure shows a reference body placed into the loading device. The bottom displacement of the top plate is touched by a steel rod, which transfers this movement directly to the laser interferometer. The spring is necessary to have the rod in touching contact to the reference body's bottom. With different material and/or thickness of the top plate different stiffness of these bodies can be achieved.

on $5 \mathrm{~mm}$ high trabecular bone cores in the culture-loading chamber we observed apparent stiffness of these cores in a range of 40 (elderly human) to $900 \mathrm{MPa}$ (bovine) under $25 \mu \mathrm{m}(4,000 \mu \mathrm{e})$ of compression. Figure 7 shows the stress/strain curves of several bone cylinders that show typical mechanical properties.

Based on these values, seven reference bodies were constructed from aluminium and stainless steel, which were specifically designed to mimic the MPa range observed in the bovine bone cores. The reference bodies were designed in the general form of a closed hollow cylinder, open at one end so that the deformation occurring at the bottom of the end plate could be measured by an interferometer during the application of a known force (Figure 8 ). The thickness of the top plate, made from either stainless steel or aluminium, was calculated based on a specific stiffness and machined to those specifications. The stiffness range (Young's modulus) of the seven reference bodies was found in the range of $40-1700 \mathrm{MPa}$ as shown in Table 1. The reference bodies were identified and labelled by the material used and the thickness of the top plate. For example: reference body "Steel 800 " has a topplate made of steel with a thickness of $800 \mu \mathrm{m}$.

The upper part of these bodies has the same dimension as the standard loading piston (see Figure 1d). The total height of the reference bodies was $5 \mathrm{~mm}$ (height of bone cores) plus the height of the piston. The termination at the bottom is identical to our standard bone chamber bottom but with a hole in the middle for passage of the steel rod to transfer the movement of the bottom of the end plate to an electronic Laser-interferometer (type UPM, made by Intop-Precision, Polytec, Germany) (Figure 8). The interferometer operates in a digital mode (counting mode) and in an additional analogue mode (homodyne mode). This allows a resolution in the sub-wavelength range. The resolution is $10 \mathrm{~nm}$ and noise mostly is $\pm 10 \mathrm{~nm}$. A built-in temperature sensor eliminates the small wavelength shift of the laser-diode at different ambient temperatures.

The reference bodies permit measurements that are very reproducible. Due to the design of theses bodies the movement of the bottom of the top plate should represent the displacement of bone cores, the deformation under the loading piston.

To find the relation between deformation under the loading piston and piezo-actuator's expansion for the reference bodies, we used quasi-static loading. With a preload of always $2.5 \mathrm{~N}$ (to bring all surfaces into touching contact) we measured displacement (d) (interferometer's data), piezo-electric actuator's expansion ( $\mathrm{x}$ ) and the force (f) in all seven reference bodies 8 times each. Depending on the stiffness of a reference body we recorded approximately 300 data triplets (f, $\mathrm{x}, \mathrm{d})$ per repetition and per body (7x8x300 data triplets). 


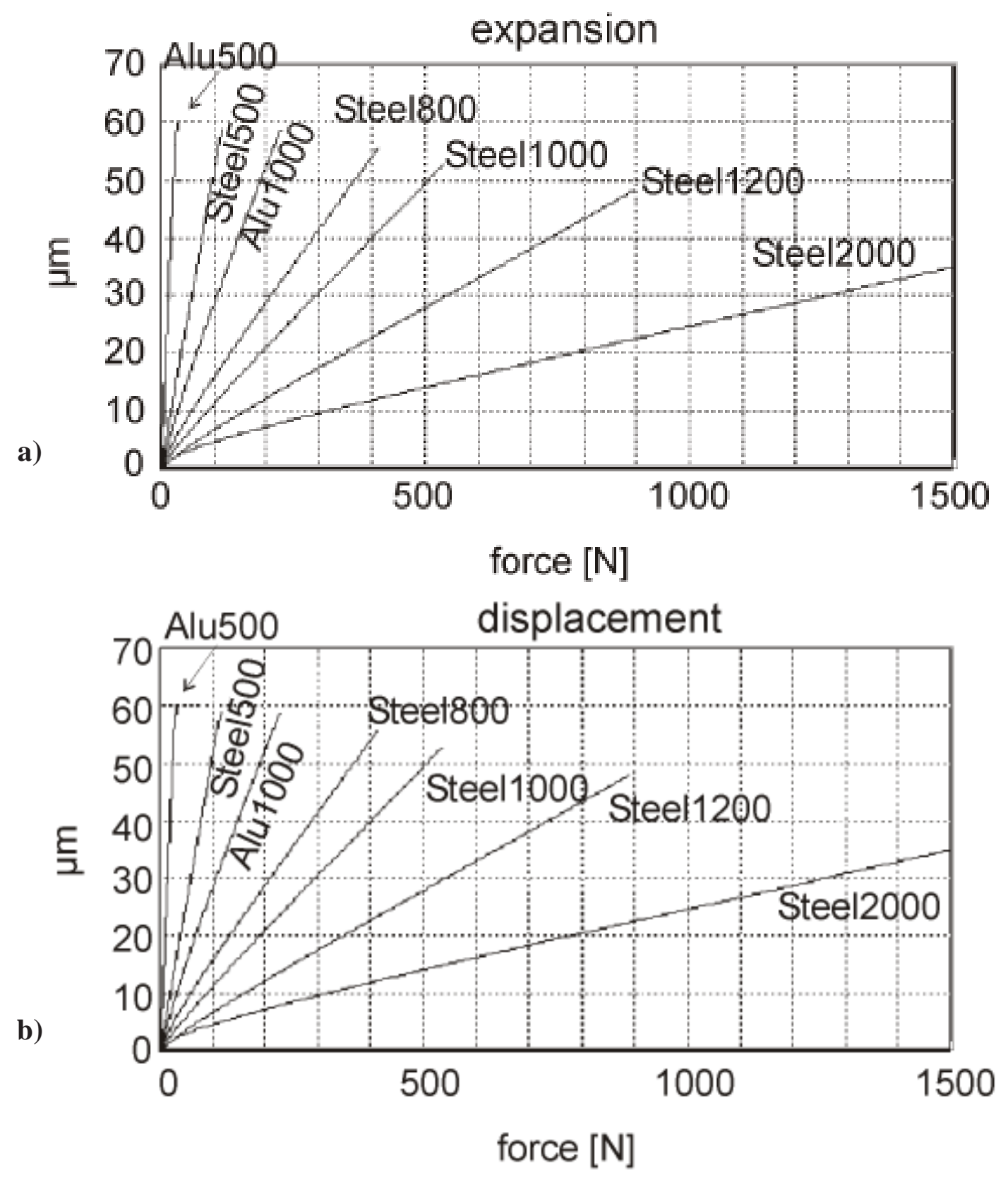

Figure 9: (a) Measurements on reference bodies: mean value of 8 repeats: expansion versus Force. (b) Measurements on reference bodies: mean value of 8 repeats: displacement versus Force. Displacement was measured by the laser interferometer

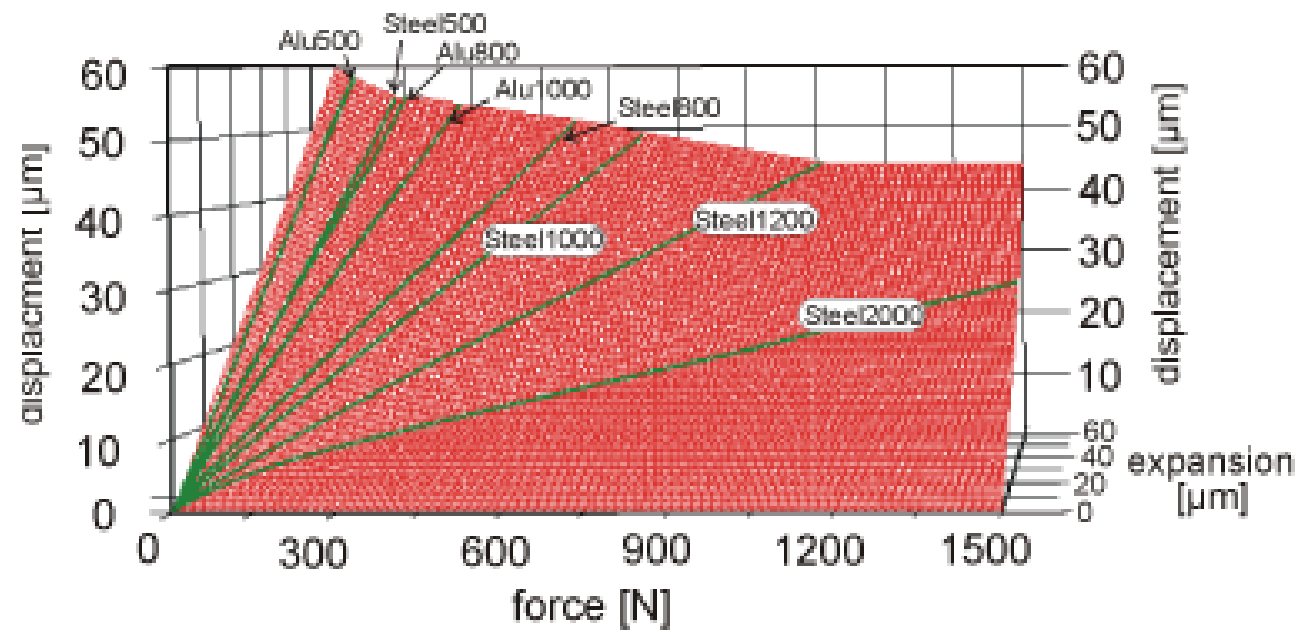

Figure 10: The data of the curves shown in figure $9 \mathrm{a} / 9 \mathrm{~b}$ are used to create a look-up table. Digital values of force (f) and expansion (x) are used to address the horizontal f,x-plane. The correct displacement is taken from the vertical axis. 


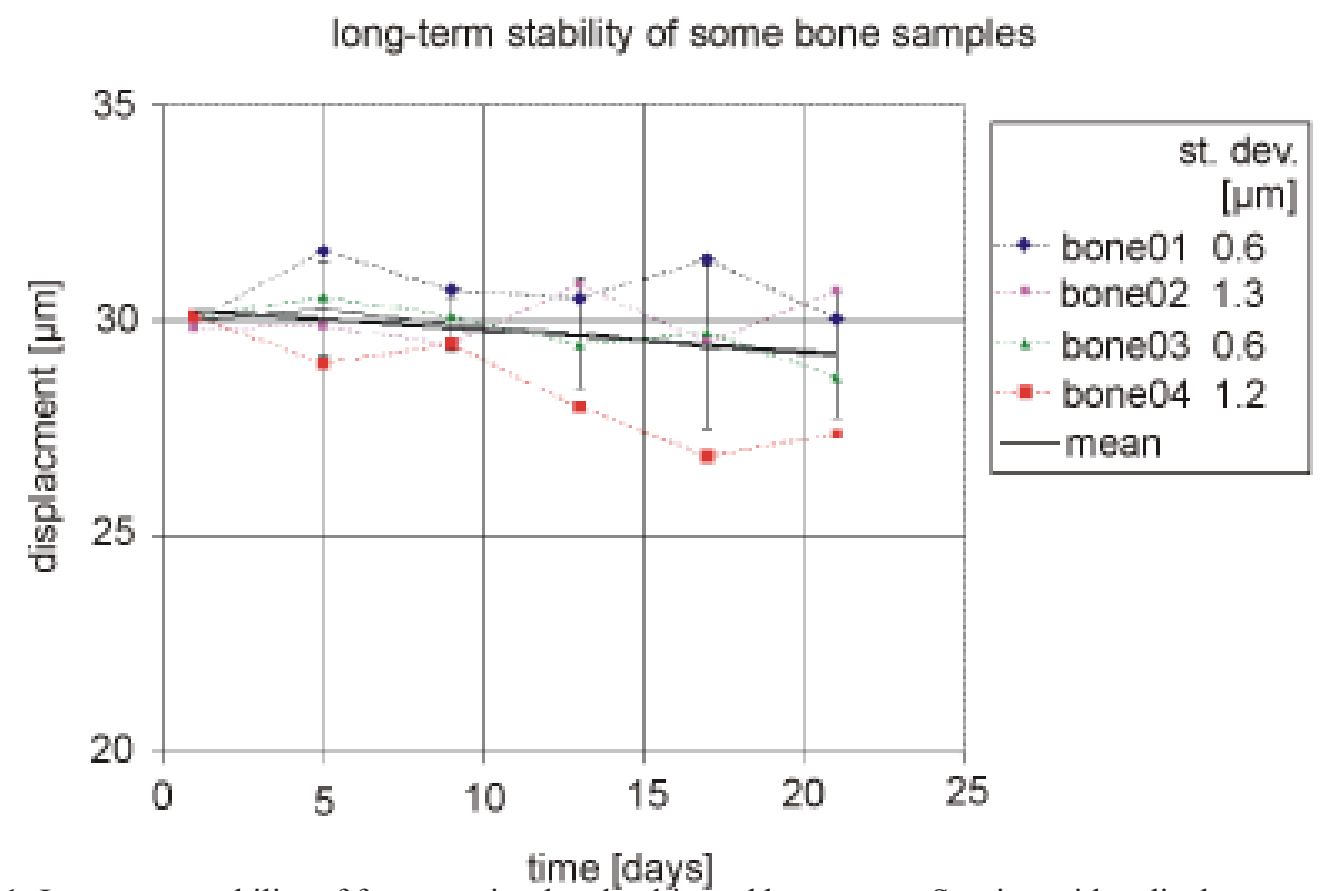

Figure 11: Long-term stability of four un-stimulated cultivated bone cores. Starting with a displacement of $30 \mu \mathrm{m}$ at constant force the bone samples were measured over 21 days every $4^{\text {th }}$ day. There is also small tendency of decreasing stiffness.

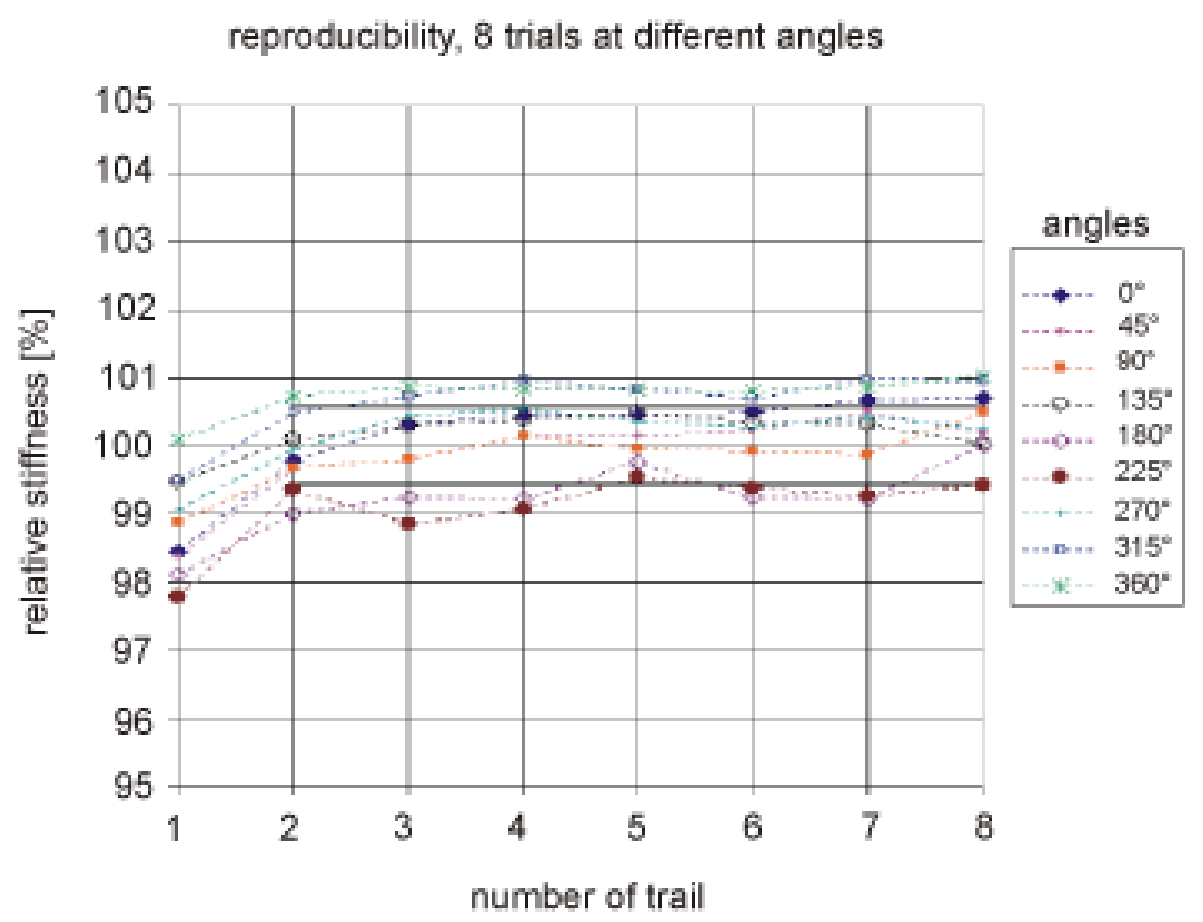

Figure 12: This figure shows 10 measurements of the stiffness of one reference body. This was done at 8 different rotation angles. Thus we obtained 8 curves. The standard deviation $0.6 \%$ (two horizontal thick lines) is used to express the reproducibility. Data of the first trial were discarded (see text).

\begin{tabular}{|c|c|}
\hline $\begin{array}{c}\text { reference } \\
\text { Body }\end{array}$ & $\begin{array}{c}\text { simulated Young's } \\
\text { modulus }\end{array}$ \\
\hline & {$[\mathrm{MPa}]$} \\
\hline & $\approx 40$ \\
\hline Alu 500 & $\approx 120$ \\
\hline Steel 500 & $\approx 240$ \\
\hline Alu 1000 & $\approx 460$ \\
\hline Steel 800 & $\approx 560$ \\
\hline Steel 1000 & $\approx 900$ \\
\hline Steel 1200 & $\approx 1700$ \\
\hline Steel 2000 & \\
\hline
\end{tabular}

Table 1. Simulated Young's Modulus of Reference Bodies These values are estimated values only, because the displacement-force relation is non-linear. Linearity is not necessary; the calibration procedure does not require Hook's bodies.

\begin{tabular}{|c|c|cc|}
\hline stiffness range & low force range & high force range \\
\hline (Y-modulus range) & below 400N & above 400N \\
\hline$[\mathrm{Mpa}]$ & & & \\
\hline $1-100$ & $+1 \mu \mathrm{m} \quad 1.6 \%$ & $+1 \mu \mathrm{m}$ & $1.6 \%$ \\
\hline $100-1000$ & $+0.5 \mu \mathrm{m} 0.8 \%$ & $+1 \mu \mathrm{m}$ & $1.6 \%$ \\
\hline$>1000$ & $+1.5 \mu \mathrm{m} 2.5 \%$ & $+2 \mu \mathrm{m}$ & $3.3 \%$ \\
\hline
\end{tabular}

Table 2. Displacement Variations

These values present a summary of the standard deviation of 8 successive displacement measurements of all reference bodies 
what would be expected in a sedentary condition over this period.

The "steel 1200" stainless steel reference body was measured for stiffness by quasi-static load every day over 31 days. The $\left[f_{a}, f_{b}\right]$ force range of 500 to $900 \mathrm{~N}$ was used for all 31 days. The mean Young's modulus of the reference body was 941.29 MPa with SD of 13.23 MPa, which is $1.4 \%$.

The same reference body was measured for stiffness 8 -times (quasi-static load) with incremental rotation at $45^{\circ}$ to determine position dependency due to possible either the chamber base or the loading-frame platform not being precisely flat and parallel. We found a relative standard deviation of $0.6 \%$ (Figure 12).

In comparison with the long-term measurement there is no significant rotation-effect in bone samples as we expected since the X-ring eliminates tilting around the vertical axis. However all curves shown in Figure 12 show another effect: There was an apparent significant increase of stiffness (trial 1 to trial 2), between days 1 and 2, which could be caused by position setting effects (touching area adaptation). We find a general increase in apparent stiffness of about $3 \%$ between culture days 1 and 2 (i.e. after the first period of loading). This can also occur with plastic and metal standards as we think that this is a mechanical adaptation to the chamber. We therefore use the $2^{\text {nd }}$ days measurement as the baseline.

\section{Preload Effects}

Due to the nature of the complex loading device and the numbers of surfaces that touch, a preload is necessary to bring all these surfaces into contact. There are five contact surfaces: PZA - piston, piston - bone, bone - top of bone chamber base plate, bottom of bone chamber base plate - frame and the helical contact surface of the position thread (Figure 1).

Based on the long-term stability data (Young's modulus of a reference body) and those of the PZA measurements on bone cores, it was determined that for long-term reproducibility a manually applied preload should be about $10 \mathrm{~N}$ and some micrometers displacement which were applied by PZA's expansion under the control of the program. We would expect some roughness at the surfaces of cut bone cores, which would, under light loads appear non-linear. We decided to use an additional preload of 5 micrometer, which is approximately the double the estimated roughness our bone surfaces (two surfaces: top and bottom) and will be absorbed by these weak (and dead) structures. Lower preloads cause loss of reproducibility and linearity.

In dynamic bone stimulation the bone was first exposed to a dynamic waveform (this period can last for some minutes) and then the stiffness was measured with a quasi-static load afterwards. One reason for this is the visco-elastic effect, in which the bone relaxes quickly and looses up to $20 \%$ loss of stiffness, and recovers by one hour

\section{Discussion}

In this paper we demonstrate the design and performance of a mechanical loading system that works with extremely high precision on small but accurately machined cylinders of trabecular bone $10 \mathrm{~mm}$ diameter and $5-10 \mathrm{~mm}$ in height. The frame, the microcontroller design, the microcontroller software and the Windows software have been designed in-house. The system is designed primarily to apply precise defined compressive strains to living trabecular bone samples, which are principally loaded in compression in vivo. Some mechanical properties of the tissue may be measured but this is not the principle aim of the device since the measurements are not presently correlated with the structure. The instrument has some significant advantages over other mechanical testing instruments used to load and test living bone specimens, not only because of the high precision, but also in the frequency spectrum it can or potentially can address. Using histology sectioning saws the samples can be machined to the required high precision, which reduces the pre-load stress needed to make accurate mechanical measurements and ensures that physiological levels of compression can be applied to the tissue. Typically $5-10 \mathrm{~N}$ only are required to achieve this, which corresponds to a maximum theoretical possible strain (assumption: $500 \mathrm{MPa}$, linear body - Hook's law) of around 3-7\% of physiological strain we use $(3,000-4,000 \mu \mathrm{e})$. Preliminary studies indicate that due to the preparation, the margins of the bone to a depth of about $500 \mu \mathrm{m}$ have a significant number of dead cells. Thus the small surface roughness of $\pm 2 \mu \mathrm{m}$, which will experience higher compressions, is probably not affecting the results by receiving high strain. Preliminary results with histology do not indicate any fractures at the bone-piston interface after 49 days of loading, or any fractures at all within the sample. Since the surfaces of the bone are the exchange surfaces for diffusion we cannot improve mechanical contact by using for instance a hard cement at the loading interfaces. In a separate paper we will present data to show the effects of amplitude and frequency on changes in bone stiffness. When combined with long term culture of living bone or cartilage the range of possible studies is large. To adapt the system for measurements on softer cartilage a piezo-electrical actuator with a large expansion range should be used. Actuators with extensions up to $180 \mu \mathrm{m}$ are available, higher extensions require a lever mechanism.

\section{Acknowledgements}

We wish to thank the European Space Agency Microgravity Applications Program (AO 99-122), the AO ASIF, Davos (grants AORF 200J and O2 J7) and the Deutsche Arthrose Hilfe for supporting this work.

\section{Patents}

United States Patent $6^{\text {th }}$ July, 1998, No.: US 6,171,812: Everett L. Smith, David Jones: Combined perfusion and mechanical loading system for explanted bone. 
United States Patent $19^{\text {th }}$ March 2002, No.: US 6,357,303 B2: Everett L. Smith, David Jones: Mechanical testing device.

\section{References}

Akhter MP, Cullen DM, Recker RR (2002) Bone adaptation response to sham and bending stimuli in mice. $\mathrm{J}$ Clin Densitom 5: 207-216.

Botchwey EA, Pollack SR, Levine EM, Laurencin CT (2001) Bone tissue engineering in a rotating bioreactor using a microcarrier matrix system. J Biomed Mater Res 55: 242-253.

Cheng MZ, Zaman G, Rawlinson SC, Suswillo RF, Lanyon LE (1996) Mechanical loading and sex hormone interactions in organ cultures of rat ulna. J Bone Miner Res 11: 502-511.

Chow JW, Wilson AJ, Chambers TJ, Fox SW (1998) Mechanical loading stimulates bone formation by reactivation of bone lining cells in 13-week-old rats. J Bone Miner Res 13: 1760-1767.

El Haj AJ, Minter SL, Rawlinson SC, Suswillo R, Lanyon LE (1990) Cellular responses to mechanical loading in vitro. J Bone Miner Res 5: 923-932.

Jones DB, Leivseth G, Tenbosch J (1995) Mechanoreception in osteoblast-like cells. Biochem Cell Biol. 73: 525-534.

Rawlinson SC, Mosley JR, Suswillo RF, Pitsillides AA, Lanyon LE (1995) Calvarial and limb bone cells in organ and monolayer culture do not show the same early responses to dynamic mechanical strain. J Bone Miner Res 10: $1225-1232$.

Rodan GA, Mensi T, Harvey A (1975) A quantitative method for the application of compressive forces to bone in tissue culture. Calcif Tissue Int 18: 125-131.

Rubin CT, Lanyon LE (1997) Kappa Delta Award paper. Osteoregulatory nature of mechanical stimuli: function as a determinant for adaptive remodeling in bone. $\mathrm{J}$ Orthop Res 5: 300-310.

Rubin CT, Turner AS, Muller R, Mittra E, McLeod K, Lin W, Qin YX (2002) Quantity and quality of trabecular bone in the femur are enhanced by a strongly anabolic, noninvasive mechanical intervention. J Bone Miner Res 17: 349-357.

Smith EL, Martens F, Koller K, Clark W, Jones DB (2000) The Effects of 20 Days of Mechanical Loading Plus PTH on The E-Modulus of Cow Trabecular Bone. J Bone Miner Res 15(Suppl 1): 247

Smith EL, Boudriot U, Daume B, Kratz M, Jones DB, Cullen D (2001) Long Term Perfusion Loading of Trabecular Bone Cores and Formation Rate. J Bone Miner Res 16(Suppl 1): 195.

Yeh CK, Rodan GA (1984) Tensile forces enhance prostaglandin $\mathrm{E}$ synthesis in osteoblastic cells grown on collagen ribbons. Calcif Tissue Int 36 (Suppl 1): 67-71

Zaman G, Suswillo RF, Cheng MZ, Tavares IA, Lanyon LE (1997) Early responses to dynamic strain change and prostaglandins in bone-derived cells in culture. J Bone Miner Res 12: 769-777

\section{Discussion with Reviewers}

J. Gasser: The authors point out that the purpose of determining bone stiffness is not to get the 'real' value but to be able to look at relative changes over time. While the reason they give for this desire (disrupted architecture at the periphery of the cores) is understandable, it could be argued, that as they wish to run finite element analysis on some of the cores, it would indeed be nice to get as close as possible to the 'real' value for stiffness.

Authors: Can we ever get the 'real' value for stiffness when we cut out a piece of bone? We think not. Firstly we considered the problem of lack of struts as we cut out the core from its mechanical surroundings and secondly we consider the supporting effect of the marrow. In a 'real' piece of bone we would expect the marrow not to be squeezed out easily into free space. The trabecular bone is constrained not only by the marrow but also by a thin shell of cortical bone which the marrow will also push against. We do not assume that our piece of bone is weaker because there can be non linear effects in spring systemsbut different. Hence we prefer to look at changes in relative stiffness and test that this is a biological response (data to be published).

J. Gasser: Authors mention the damage caused to the outer $500 \mu \mathrm{m}$ of the cores during manufacturing. Is this inflicted by the heat generated during cutting? In a core of 5 to $10 \mathrm{~mm}$ height does it worry authors that as much as $20 \%$ of the structure they try to stimulate is dead and possibly unable to respond to the mechanical or chemical stimuli?

Authors: Does it worry us that perhaps $20 \%$ is dead? Yes! What to do about it. We are cutting the bone at about $8^{\circ} \mathrm{C}$ in PBS. We think that the surface layer of cells is damaged more from too fast cutting and heat, but other cells are dying due to the phenomenon known as neighbour death. It should be possible to use inhibitors of apoptosis to reduce this, but it can cost a lot of money. It is difficult for several reasons to establish cell death in bone. In some preparations we see living cells at the surface, so it is something we are looking at.

J. Gasser: The authors point out the many possibilities the system offers to study living bone or cartilage. Please be more specific in what you want to study with this system and as pointed out earlier, add a comparison as to where you think the system is performing better than currently available organ culture systems and where you think it is not coming close to the in vivo situation (shortcomings).

Authors: The system was designed to do three things- (1) apply all sorts of mechanical waveforms- frequencies and amplitudes - not necessarily physiological to investigate mechano-transduction in bone and cartilage, (2) ensure the mechanical properties of the biological material, also with regard to the non linearity and visco elastic behaviour, (3) Maintain the material in as good a condition as possible. The answer as to how this system is better or worse than other organ culture systems is not described 
here, but will be presented for publication soon. Very briefly what the system does not have is functioning blood vessels or nerves. The lack of blood vessels should have proved a problem, but practically we see no significant changes in the histology of the marrow or bone until over 50 days (this data will be published soon), which is something no other bone organ culture system can do.

P. Prendergast: Looking at the quasi-static load measurement $\sin$ in Figure 7, I do not understand why they do not start at 0,0 load/displacement? Have the measurements made in the device been corroborated with measurement made in a materials testing machine?

Authors: We are working with dimensions in the tens of micrometer range of compression. All the mechanical parts that can be are pre-compressed, but there are still many mechanical surfaces which are not perfectly flat (meaning here an optically flat surface less than $1 / 2$ lambda). Thus these and the inevitable inaccuracies of cutting the bone cylinders mean that we have to apply a certain preforce to 'mate' all these surfaces. We calculated that a surface roughness of roughly 4 micrometers would need a pre-force of about 1/10th of the ultimate force we would apply-assuming the stiffness of the bone cores would need about $100 \mathrm{~N}$ of force to achieve a compression of 3,000 microstrains, i.e. $1 / 10$ th of a supposedly stimulatory signal. In actuality we can sometimes make measurements on bone with an error of less than 5 percent or a pre force of $5 \mathrm{~N}$. This seems to depend on the material properties of the bone under test. If we made the bone cores $10 \mathrm{~mm}$ long (which is possible using the present design) we could in theory make 'better' mechanical measurements, however we have not yet tested this in terms of how the bone will react to the longer diffusion paths. These studies are planned for the future and will be addressed in another paper.

P. Prendergast: The calibration with the reference bodies is interesting, though I wonder what biologists reading the journal will make of it. I do not think the "lookup" table of Figure 10 helps much and I suggest that it should be deleted. The reference bodies are all relatively stiff, which tests for the internal deformations within the mechanisms of the device when loading stiff samples. However, presumably the displacement with stiff reference bodies is low -have you tried a flexible reference body where the displacement may be larger to assess reproducibility?

Authors: We chose the stiffness of the reference bodies to match the range of stiffness we were measuring. Because no other material or (materials) was conveniently available over this range we manufactured them and calibrated them as described. The use of the interferometer gave us an as accurate measurement of displacement as we could hope for. We indeed tested the results several times against commercially available ones such as Dartec or Instron, and also using solid reference bodies of aluminium, which have been measured in other devices, but against not more sensitive machines ones which are available. The results showed that our machine when properly calibrated is as accurate as shown in the paper. 